\title{
Patient-specific pluripotent stem cell-based Parkinson's disease models showing endogenous alpha-synuclein aggregation
}

\author{
Yohan $\mathrm{Oh}^{1,2, *}$ \\ ${ }^{1}$ Department of Medicine, College of Medicine, Hanyang University, ${ }^{2}$ Graduate School of Biomedical Science and Engineering, Hanyang \\ University, Seoul 04763, Korea
}

\begin{abstract}
After the first research declaring the generation of human induced pluripotent stem cells (hiPSCs) in 2007, several attempts have been made to model neurodegenerative disease in vitro during the past decade. Parkinson's disease (PD) is the second most common neurodegenerative disorder, which is mainly characterized by motor dysfunction. The formation of unique and filamentous inclusion bodies called Lewy bodies (LBs) is the hallmark of both PD and dementia with LBs. The key pathology in PD is generally considered to be the alpha-synuclein ( $\alpha$-syn) accumulation, although it is still controversial whether this protein aggregation is a cause or consequence of neurodegeneration. In the present work, the recently published researches which recapitulated the $\alpha$-syn aggregation phenomena in sporadic and familial PD hiPSC models were reviewed. Furthermore, the advantages and potentials of using patient-derived PD hiPSC with focus on $\alpha$-syn aggregation have been discussed. [BMB Reports 2019; 52(6): 349-359]
\end{abstract}

\section{INTRODUCTION}

Parkinson's disease (PD) is a progressive, age-related neurodegenerative disease with noteworthy motor impairments, and is the second most common neurodegenerative disease after Alzheimer's disease (AD). PD is primarily linked with the explicit loss of midbrain dopaminergic (mDA) neurons in the substantia nigra pars compacta (SNpc), and physically displays as weakened movements in affected individuals $(1,2)$. The formation of unique and filamentous inclusion bodies called Lewy bodies (LBs), comprised mostly of alpha-synuclein ( $\alpha$-syn, SNCA gene product), is considered as the hallmark of PD or dementia with LBs (DLB) (1-6). Although the key

${ }^{*}$ Corresponding author. Tel: +82-2-2220-2423; Fax: +82-2-22202422; E-mail: yoh@hanyang.ac.kr

https://doi.org/10.5483/BMBRep.2019.52.6.142

Received 17 April 2019

Keywords: Aggregation, Alpha-synuclein, Disease modeling, hiPSC, Parkinson's disease pathology in PD or DLB is commonly known to be the accumulation of misfolded- and aggregated- $\alpha$-syn (2-7), the formation of pathological $\alpha$-syn aggregates is not typically displayed in general neurotoxin-based PD animal models (8, 9). In addition, several trials for PD drugs continue to fail; this leads to a significant socioeconomic burden on our healthcare system and emphasizes the need for a new approach to model PD pathogenesis.

Human pluripotent stem cells (hPSCs), including embryonic stem or induced pluripotent stem cells (hESCs/hiPSCs), differentiated into specific types of neurons have emerged as a promising model for studying human neural diseases (10-14) and have the potential to be used as cell sources for transplantation $(15,16)$. Particularly, disease-specific hiPSCs provide us with an exceptional opportunity to recapitulate human disease phenotypes in vitro, thereby enabling disease investigation and drug development; although there are several challenges which need to be addressed [reviewed in (17)]. Using PD patients' hiPSCs, many researchers have tested whether PD-relevant phenotypes are reproduced in the patient-derived mDA neurons in vitro to reveal PD pathology and to identify therapeutic targets; however, abnormally phosphorylated detergent-insoluble $\alpha$-syn aggregates are rarely recapitulated in these model systems [reviewed in $(18,19)]$.

In this review, it was investigated whether the $\alpha$-syn aggregation phenomena in PD or DLB can be reproduced in hiPSC-based models, the present extent of development, and the type of further researches necessitated in future.

\section{$\alpha$-SYNUCLEIN AGGREGATION AND $\alpha$-SYNUCLEINOPATHY}

At the time of initial cloning, $\alpha$-syn was called as the 'precursor of non-A $\beta$ component of AD amyloid' (precursor of NAC, NACP) because the NAC was first detected in and isolated from $A D$ amyloid plaques (20). $\alpha$-Syn protein is a soluble protein and exists in the form of an unfolded monomer (21). Furthermore, it has been considered that $\alpha$-syn undergoes a conformational change to the $\alpha$-helical structure only upon binding to lipid vesicles (22). As unstructured $\alpha$-syn monomers tend to eagerly undergo a conformational change to $\beta$-sheet structure and aggregate together, it has been hypothesized that 
binding of lipid vesicles with the $\alpha$-helical conformation of $\alpha$-syn monomers is a crucial intrinsic mechanism for sequestering unfolded cytosolic $\alpha$-syn to prevent spontaneous $\alpha$-syn aggregation. However, recent studies indicate that $\alpha$-syn exists in the form of an $\alpha$-helically folded tetramer in the physiological conditions and not as a natively unfolded monomer, and rarely this tetramer is converted into the pathological aggregates $(23,24)$. The PD missense mutations located in the lipid-binding motif of $\alpha$-syn increase the transition from tetramer to monomer (25), leading to the formation of $\beta$-sheet oligomers and eventually to insoluble aggregates in pathological conditions. A growing number of evidence indicates a causative role of $\alpha$-syn misfolding and aggregation in the pathogenesis of PD $(2-7,26,27)$. $\alpha$-Synucleinopathies (also called synucleinopathies) are neurodegenerative diseases representing the abnormal accumulation of intracellular aggregates of $\alpha$-syn in neurons (LB and Lewy neurite) or glial cells (28). Among $\alpha$-synucleinopathies, PD, DLB, and multiple system atrophy (MSA) are of the most common occurrence. The incident rate of $\alpha$-synucleinopathies related to parkinsonism was 21.0 per 100,000 person-years (PD, $68 \%$ of $\alpha$-synucleinopathies; DLB, $28 \%$; MSA, $4 \%$ ), based on the investigation of the medical records in Olmsted County, Minnesota, USA, 1991-2005 (29). Classical neurotoxin-based animal models do not model the molecular pathology of $\alpha$-synucleinopathies $(8,9)$. However, many attempts have been made to recapitulate $\alpha$-synucleinopathies in the mammalian system using $\alpha$-syn transgenic mouse models, viral vector models of $\alpha$-syn overexpression, and $\alpha$-syn transmission models [reviewed in (9)].

\section{PHOSPHORYLATION OF $\alpha$-SYNUCLEIN}

Phosphorylation is the most widely and deeply studied posttranslational modification of $\alpha$-syn. This modification may affect the ability of aggregate formation as well as the subcellular localization and function of $\alpha$-syn. In recent years, an increasing number of studies have reported that $\alpha$-syn within LBs is subjected to phosphorylation at serine 129 (S129), and it may have serious implications for $\alpha$-syn-induced neurodegeneration (7, 30-34). Especially, S129-phosphorylated $\alpha$-syn (pS129- $\alpha$-syn) is an excellent marker for $\alpha$-synucleinopathies because $\sim 90 \%$ of $\alpha$-syn in LBs is phosphorylated at S129, compared with only $\sim 4 \%$ of $\alpha$-syn under physiological conditions (7). However, the molecular and cellular mechanisms of $\alpha$-syn aggregation controlled by phosphorylation and other effects of $\alpha$-syn phosphorylation at S129, remain to be elucidated $(35,36)$, probably due to a lack of a pathophysiologically relevant model system for investigating $\alpha$-syn aggregation of $\alpha$-synucleinopathies. Therefore, it should be clearly addressed whether $\alpha$-syn phosphorylation is a cause or a consequence of aggregation, or whether phosphorylation is neurotoxic or neuroprotective in $\alpha$-synucleinopathies including PD and DLB.

\section{HUMAN PLURIPOTENT STEM CELL-DERIVED MIDBRAIN DOPAMINERGIC NEURONS}

Developing the most ideal protocol for human mDA neuronal differentiation from hPSCs for applications in PD modeling and/or transplantation therapy has been an intense area of research during the past decade. Arenas et al. have reviewed the molecular mechanisms underlying $\mathrm{mDA}$ neuronal development in vivo and their applications for in vitro generation of human $\mathrm{mDA}$ neurons differentiated from hPSCs or directly induced from somatic cells [reviewed in (37)]. In this section, popular and major protocols for the human $\mathrm{mDA}$ neuronal differentiation from hPSCs are briefly introduced (Fig. 1).

\section{Stromal feeder-induced midbrain dopaminergic neurons}

The classical approach to developing a protocol for the human mDA neuronal differentiation from hPSCs was based on adaptations of mouse neural stem cell (mNSC) and mESC protocols, which required co-culture with feeder cells (37). Three groups have published the initial protocols to derive mDA neurons from hESCs co-cultured with the murine stromal cell lines (38-40). In 2007, Sonntag et al. reported an enhanced protocol to generate neural rosette-derived $\mathrm{mDA}$ neurons from hESCs co-cultured with the MS-5 cells as a feeder, appropriate for cell therapy in PD (41) (Fig. 1A).

\section{Embryonic body-derived midbrain dopaminergic neurons} For initiating spontaneous differentiation from hPSC towards specific cell types, embryonic body (EB)-formation is often considered as a basic starting method. After inducing EB-formation, putative $\mathrm{mDA}$ neurons are generated from hESCs $(42,43)$. In 2009, Swistowski et al. published a protocol to derive expandable $\mathrm{mDA}$ progenitors and $\mathrm{mDA}$ neurons from $\mathrm{hESCs}$ via $\mathrm{EB}-$ formation and neural rosette-isolation (44) (Fig. 1B)

\section{Floor plate-derived midbrain dopaminergic neurons}

Studer group introduced a "dual-SMAD inhibition" method for differentiating hPSCs into neural cells in an exceedingly efficient manner to eliminate the influence of undefined factors, including unknown secreted molecules and unidentified effects of co-culturing with murine stromal cell lines or astrocytes, as well as to increase the efficiency and to reduce heterogenous nature on neuronal differentiation (45). Combined blockage of SMAD signaling at the beginning of the monolayer differentiation protocol using Noggin (to inhibit BMP-mediated SMAD signaling) and SB431542 (to inhibit TGF- $\beta /$ nodal/activin-mediated SMAD signaling), synergistically facilitated neural induction of hPSCs and eliminated the need for feeder layers (45).

The developing midbrain co-expresses the roof plate marker LMX1A and the floor plate (FP) marker FOXA2. Administration 


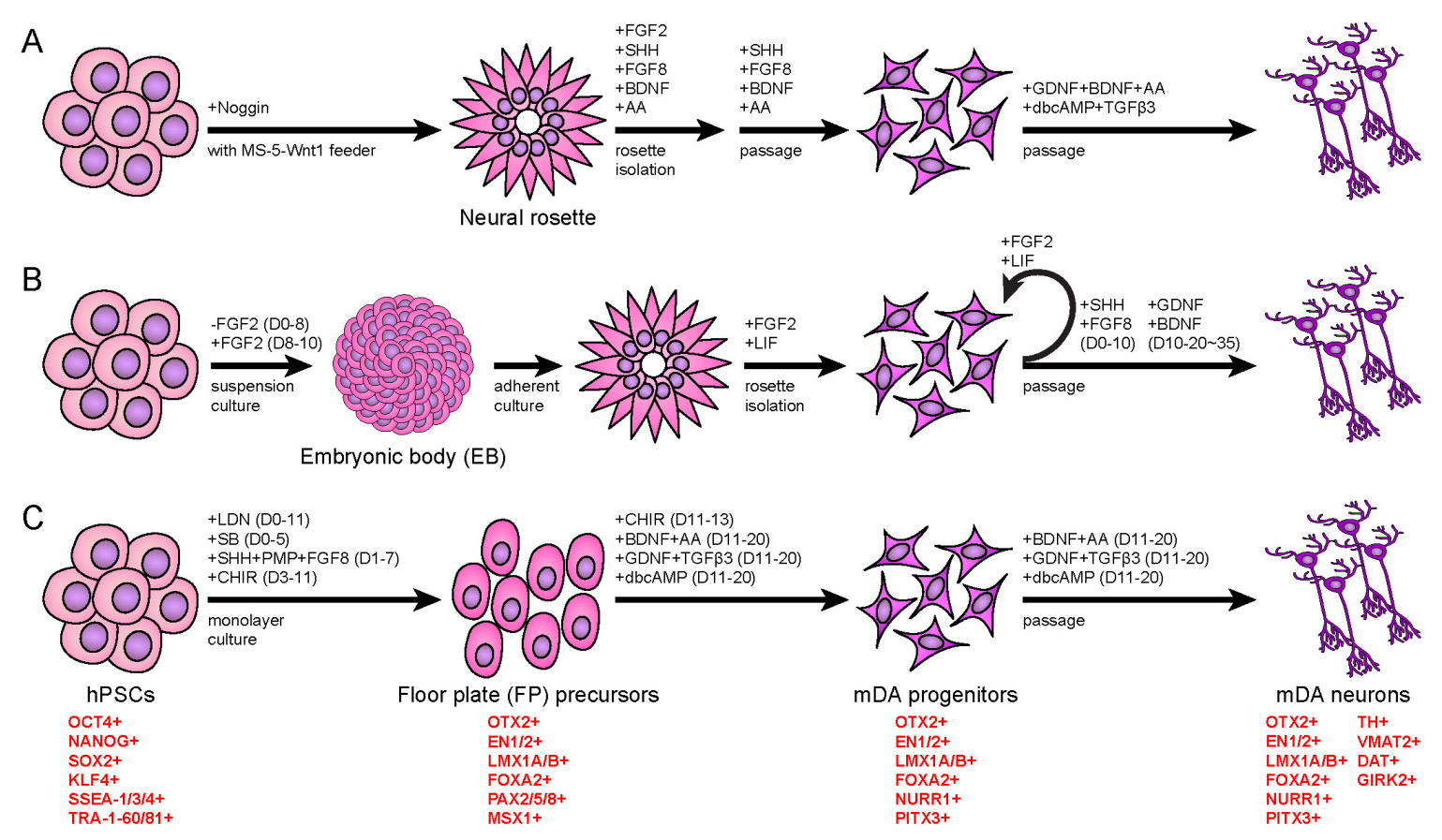

Fig. 1. Derivation of human midbrain dopaminergic neurons from hPSC. (A) A protocol developed by Sonntag et al. for the generation of human mDA neurons via the neural rosette-isolation, from hESCs co-cultured with the MS-5 cells as a feeder (41). (B) A protocol developed by Swistowski et al. for the generation of expandable mDA progenitors and mDA neurons from hESCs via the embryonic body (EB)-formation and neural rosette-isolation (44). (C) A protocol developed by Kriks et al. for the generation of hPSC-derived mDA neurons via the floor plate (FP)-induction (47). AA, ascorbic acid. CHIR, CHIR99021. dbcAMP, dibutyryl-cyclic AMP. LDN, LDN193189. MS-5-Wnt1, Wnt1 transgenic MS-5 cells. PMP, purmorphamine. SB, SB431542.

of high levels of $\mathrm{SHH}$ along with the dual-SMAD inhibition during neural induction has been considered as essential for FP specification (46). By synthesizing the existing knowledge of midbrain development, Studer group succeeded in generating correctly specified hPSC-derived mDA neurons in a reliable and efficient manner (47). A follow-up study has described the use of a small molecule, LDN193189 (to inhibit BMP-mediated SMAD signaling), that can replace Noggin for neural induction of hPSCs (48). Modified dual SMAD inhibition (termed "LSB" for two inhibitors LDN193189 and SB431542) along with activation of SHH and WNT signaling, enhances the efficiency and reproducibility of the monolayer differentiation of hPSC-derived mDA neurons via FP-induction.

\section{GENETIC MODELS USING PATIENT-DERIVED HUMAN INDUCED PLURIPOTENT STEM CELL}

Jaenisch group reported the first example of PD modeling using PD patient-derived hiPSC-based DA neurons in 2009, but the focus of the study did not cover the phenotypical differences between patient's cells and healthy controls (49). After the pioneer study, many groups attempted to model PD in vitro using patient hiPSC-derived neuronal cells and reported various in vitro phenotypes consistent with PD pathophysiology [reviewed in $(18,19)$ ]. While tons of studies failed to repeat the aggregation of $\alpha$-syn in PD hiPSC-based models, a handful of researches recapitulated $\alpha$-syn aggregation in PD patient-derived hiPSC-based neuronal models by employing unique conditions (Table 1). It is necessary to state that cases showing simple augmentation of $\alpha$-syn expression were intentionally omitted in the present review.

\section{Sporadic PD hiPSC-based model}

The causes of sporadic PD and DLB are largely unknown; however, the aggregation of $\alpha$-syn is heavily implicated in the degeneration of neurons in sporadic PD and DLB (50). In 2016, Krainc group reported the detection of the thioflavin S-positive $\alpha$-syn aggregates in the FP-derived 110 days old mDA neurons, which were differentiated from a sporadic PD hiPSC (51). The authors also showed that the level of pathogenic $\alpha$-syn species detected by Syn303 antibody (52) was significantly increased in the $1 \%$ Triton X-100insoluble-and-2\% SDS-soluble fraction of the sporadic PD mDA neurons, as compared to healthy controls (51). Another report described the increased level of pS129- $\alpha$-syn in the FP-derived 28-49 days old mDA neurons differentiated from a 
Endogenous $\alpha$-syn aggregation models using PD hiPSC

Yohan Oh

Table 1. Selected reports showing the endogenous $\alpha$-synuclein aggregation using PD hiPSC-derived cell models

\begin{tabular}{|c|c|c|c|c|c|c|c|c|c|}
\hline \multirow{2}{*}{$\begin{array}{l}\text { Genetic } \\
\text { mutation }\end{array}$} & \multirow{2}{*}{$\begin{array}{l}\text { Tested } \\
\text { model }\end{array}$} & \multirow{2}{*}{$\begin{array}{l}\text { Differentiation } \\
\text { protocol (ref.) }\end{array}$} & \multirow{2}{*}{$\begin{array}{l}\text { Differentiation } \\
\text { marker }\end{array}$} & \multirow{2}{*}{ Stimulus - } & \multicolumn{2}{|c|}{ Aggregate } & \multirow{2}{*}{$\begin{array}{l}\text { Cell } \\
\text { death }\end{array}$} & \multirow{2}{*}{ Note } & \multirow{2}{*}{ Ref. } \\
\hline & & & & & WB & ICC & & & \\
\hline \multicolumn{10}{|l|}{ Sporadic } \\
\hline Unknown & $\mathrm{mDA}$ & 2D-based $(45,47)$ & n.d. & No & Yes & Yes & n.d. & $\begin{array}{l}\text { Increased detergent-insoluble } \\
\alpha \text {-syn aggregates at day } 110 ; \\
\text { thioflavin S-positive } \alpha \text {-syn } \\
\text { aggregates formation at day } \\
110 ; \text { lysosomal defects at day } \\
90\end{array}$ & (51) \\
\hline \multicolumn{10}{|l|}{ SNCA } \\
\hline $\begin{array}{l}\text { p.[Ala53Thr] } \\
;[=]\end{array}$ & $\begin{array}{l}\text { mDA } \\
\text { GABAergic } \\
\text { GLUergic }\end{array}$ & $\begin{array}{l}\text { EB-based }(43,45,49) \\
\sim 20 \% \mathrm{TH}^{+} ; \sim 25 \% \\
\text { GABA }^{+} ; \sim 20 \% \\
\text { VGLUT1 }^{+}\end{array}$ & $\begin{array}{l}\text { FOXA2, NURR1, } \\
\text { PITX3, TH, } \\
\text { MAP2, GAD67, } \\
\text { VGLUT1 } \\
\text { (qRT-PCR); TH, } \\
\text { GABA, VGLUT1 } \\
\text { (ICC) }\end{array}$ & No & n.d. & Yes & Yes & $\begin{array}{l}\text { Detection of thioflavin } \\
\text { S-positive and proteinase } \\
\text { K-resistant } \alpha \text {-syn aggregates at } \\
\text { day 50; increased } \\
\text { pS129- } \alpha \text {-synat day 50; } \\
\text { decreased neurite length at } \\
\text { day } 50\end{array}$ & (54) \\
\hline \multirow[t]{4}{*}{$\begin{array}{l}\text { p.[Ala53Thr] } \\
\quad ;[=]\end{array}$} & $\mathrm{mDA}$ & $\begin{array}{l}\text { 2D-based }(45,47) \\
\sim 73 \% \mathrm{TH}^{+} / \text {GIRK2 }^{+}\end{array}$ & $\begin{array}{l}\text { OTX2, LMX1A, } \\
\text { FOXA2, NURR1, } \\
\text { TH, GIRK2 (ICC) }\end{array}$ & No & n.d. & Yes & No & $\begin{array}{l}\text { Increased thioflavin T-positive } \\
\text { or S129-phosphorylated } \alpha \text {-syn } \\
\text { aggregates formation and ROS } \\
\text { production than genetically } \\
\text { corrected controls at day } 35\end{array}$ & (55) \\
\hline & & & & paraquat & n.d. & n.d. & Yes & $\begin{array}{l}\text { Increased apoptosis than } \\
\text { genetically corrected controls } \\
\text { at day } 35\end{array}$ & \\
\hline & & & & maneb & n.d. & n.d. & Yes & $\begin{array}{l}\text { Increased apoptosis than } \\
\text { genetically corrected controls } \\
\text { at day } 35\end{array}$ & \\
\hline & & & & rotenone & n.d. & n.d. & Yes & $\begin{array}{l}\text { Increased apoptosis than } \\
\text { genetically corrected controls } \\
\text { at day } 35\end{array}$ & \\
\hline $\begin{array}{l}\text { p.[Ala53Thr] } \\
\quad ;[=]\end{array}$ & $\mathrm{mDA}$ & 2D-based $(45,47)$ & n.d. & No & n.d. & Yes & n.d. & $\begin{array}{l}\text { Detection of thioflavin } \\
\text { S-positive } \alpha \text {-syn aggregates at } \\
\text { day } 90 ; \text { reduced aggregates } \\
\text { formation by } 758 \text { treatment at } \\
\text { day } 90\end{array}$ & (56) \\
\hline Duplication & Neuron & EB-based (82) & n.d. & No & Yes & n.d. & n.d. & $\begin{array}{l}\text { Neural rosette-derived neurons } \\
\text { co-cultured with astrocytes; } \\
\text { increased detergent-insoluble } \\
\alpha \text {-syn aggregates; increased } \\
\text { presence of } \alpha \text {-syn aggregation } \\
\text { intermediates }\end{array}$ & (57) \\
\hline Triplication & Cortical & $\begin{array}{l}\text { 2D-based (45) } \\
\sim 70 \% \text { neurons } \\
\text { activated by } \\
\text { glutamate }\end{array}$ & $\begin{array}{l}\text { Glutamate } \\
\text { response }\left(\mathrm{Ca}^{2+}\right. \\
\text { imaging) }\end{array}$ & No & n.d. & Yes & Yes & $\begin{array}{l}\text { Increased filamentous } \alpha \text {-syn } \\
\text { aggregates formation and cell } \\
\text { death than genetically } \\
\text { corrected controls at day } \\
\text { 70-90; increased NADH redox } \\
\text { index than control at day } \\
70-90\end{array}$ & (58) \\
\hline \multirow[t]{2}{*}{ Triplication } & $\mathrm{mDA}$ & $\begin{array}{l}\text { EB-based (53) } \\
\sim 28 \% \mathrm{TH}^{+} / \\
\text {TUBB3 }^{+}(83)\end{array}$ & $\begin{array}{l}\text { FOXA2, TH } \\
\text { (qRT-PCR); } \\
\text { FOXA2, TH (ICC) }\end{array}$ & No & n.d. & Yes & n.d. & $\begin{array}{l}\text { Neural rosette-derived mDA; } \\
\text { increased number and size of } \\
\text { punctate } \alpha \text {-syn aggregates at } \\
\text { day }>71 \text { than at day } 45-50\end{array}$ & (59) \\
\hline & $\mathrm{BFCN}$ & $\begin{array}{l}\text { EB-based }(84,85) \\
\sim 36 \% \mathrm{VCHT}^{+} / \\
\text {TUBB3 }^{+}(83)\end{array}$ & $\begin{array}{l}\text { CHAT (qRT-PCR); } \\
\text { CHAT (ICC) }\end{array}$ & No & n.d. & Yes & n.d. & $\begin{array}{l}\text { Neural rosette-derived BFCN; } \\
\text { increased number and size of } \\
\text { diffused } \alpha \text {-syn aggregates at } \\
\text { day }>71 \text { than at day } 45-50\end{array}$ & \\
\hline
\end{tabular}


Table 1. Continued 1

\begin{tabular}{|c|c|c|c|c|c|c|c|c|c|}
\hline \multirow{2}{*}{$\begin{array}{l}\text { Genetic } \\
\text { mutation }\end{array}$} & \multirow{2}{*}{$\begin{array}{l}\text { Tested } \\
\text { model }\end{array}$} & \multirow{2}{*}{$\begin{array}{l}\text { Differentiation } \\
\text { protocol (ref.) }\end{array}$} & \multirow{2}{*}{$\begin{array}{l}\text { Differentiation } \\
\text { marker }\end{array}$} & \multirow{2}{*}{ Stimulus } & \multicolumn{2}{|c|}{ Aggregate } & \multirow{2}{*}{$\begin{array}{l}\text { Cell } \\
\text { death }\end{array}$} & \multirow{2}{*}{ Note } & \multirow{2}{*}{ Ref. } \\
\hline & & & & & WB & ICC & & & \\
\hline Triplication & $\mathrm{mDA}$ & $\begin{array}{l}\text { 2D-based }(45,47) \\
\sim 70 \% \mathrm{TH}^{+} / \\
\text {FOXA2 }^{+}\end{array}$ & $\begin{array}{l}\text { LMX1A, FOXA2, } \\
\text { TH (ICC) }\end{array}$ & No & Yes & Yes & n.d. & $\begin{array}{l}\text { Increased detergent-insoluble } \\
\alpha \text {-syn aggregates at day } \\
55 \text {-330; thioflavin S-positive } \\
\alpha \text {-syn aggregates formation at } \\
\text { day } 100 \text { or } 120 ; \text { increased low } \\
\text { expressing TH cells at day } \\
330 ; \text { lysosomal defects at day } \\
180 \text { or } 330 ; \text { reduced } \\
\text { aggregates formation by } 758 \\
\text { treatment at day } 120\end{array}$ & $\begin{array}{r}(51, \\
56)\end{array}$ \\
\hline \multicolumn{10}{|l|}{$L R R K 2$} \\
\hline $\begin{array}{l}\text { p.[Gly2019Ser] } \\
\quad ;[=]\end{array}$ & Astrocyte & $\begin{array}{l}\text { 3D-based (86) } \\
\sim 95 \% \text { GFAP }^{+}\end{array}$ & $\begin{array}{l}\text { AQP4, GFAP } \\
\text { (qRT-PCR); } \\
\text { GFAP, S100ß, } \\
\text { GLT1 (ICC) }\end{array}$ & No & n.d. & Yes & n.d. & $\begin{array}{l}\text { Increased } \alpha \text {-syn puncta area at } \\
\text { day 28; transmitting } \alpha \text {-syn to } \\
\text { mDA neurons during } 28 \text { days } \\
\text { co-culture }\end{array}$ & (61) \\
\hline \multicolumn{10}{|l|}{$P R K N$} \\
\hline $\begin{array}{l}\text { p.[Arg42Pro] } \\
;[=]\end{array}$ & $\mathrm{mDA}$ & $\begin{array}{l}\text { EB-based (44) } \\
\sim 7 \% \mathrm{TH}^{+}\end{array}$ & FOXA2, TH (ICC) & No & u.c. & u.c. & n.d. & $\begin{array}{l}\text { Decreased mDA neuronal } \\
\text { differentiation than healthy } \\
\text { control }\left(\sim 22 \% \mathrm{TH}^{+}\right) \text {at day } \\
28\end{array}$ & (62) \\
\hline $\begin{array}{l}\text { p. }[\text { Arg275Trp }] \\
\quad ;[=]\end{array}$ & $\mathrm{mDA}$ & $\begin{array}{l}\text { EB-based (44) } \\
\sim 15 \% \mathrm{TH}^{+}\end{array}$ & FOXA2, TH (ICC) & No & u.c. & u.c. & n.d. & $\begin{array}{l}\text { Decreased mDA neuronal } \\
\text { differentiation than healthy } \\
\text { control }\left(\sim 22 \% \mathrm{TH}^{+}\right) \text {at day } \\
28\end{array}$ & (62) \\
\hline $\begin{array}{l}\text { p.[Arg42Pro] } \\
\text {;[EX3del] }\end{array}$ & $\mathrm{mDA}$ & $\begin{array}{l}\text { EB-based (44) } \\
\sim 7 \% \mathrm{TH}^{+}\end{array}$ & FOXA2, TH (ICC) & No & u.c. & u.c. & n.d. & $\begin{array}{l}\text { Decreased mDA neuronal } \\
\text { differentiation than healthy } \\
\text { control }\left(\sim 22 \% \mathrm{TH}^{+}\right) \text {at day } \\
28\end{array}$ & (62) \\
\hline $\begin{array}{l}\text { p.[Asn52fs] } \\
\quad ;[\text { EX3_4del] }\end{array}$ & $\mathrm{mDA}$ & $\begin{array}{l}\text { EB-based (44) } \\
\sim 7 \% \mathrm{TH}^{+}\end{array}$ & FOXA2, TH (ICC) & No & u.c. & u.c. & n.d. & $\begin{array}{l}\text { Decreased mDA neuronal } \\
\text { differentiation than healthy } \\
\text { control }\left(\sim 22 \% \mathrm{TH}^{+}\right) \text {at day } \\
28\end{array}$ & (62) \\
\hline $\begin{array}{l}\text { p.[Val324fs] } \\
; \text {;Val324fs] }\end{array}$ & $\mathrm{mDA}$ & $\begin{array}{l}\text { 2D-based }(45,47) \\
\sim 70 \% \mathrm{TH}^{+}\end{array}$ & $\begin{array}{l}\text { LMX1A, FOXA2, } \\
\text { NURR1, TH } \\
\text { (ICC) }\end{array}$ & No & Yes & u.c. & n.d. & $\begin{array}{l}\text { Increased Triton } \\
\text { X-100-insoluble } \alpha \text {-syn } \\
\text { aggregates at day } 60 ; \\
\text { increased intracellular } \\
\text { dopamine level; increased } \\
\text { susceptibility to mitochondria } \\
\text { toxin at day } 60\end{array}$ & (63) \\
\hline \multicolumn{10}{|l|}{ PINK1 } \\
\hline $\begin{array}{l}\text { p.[Gln456Ter] } \\
; \text {;Gln456Ter] }\end{array}$ & $\mathrm{mDA}$ & $\begin{array}{l}\text { 2D-based }(45,47) \\
\sim 75 \% \mathrm{TH}^{+}\end{array}$ & $\begin{array}{l}\text { LMX1A, FOXA2, } \\
\text { NURR1, TH } \\
\text { (ICC) }\end{array}$ & No & Yes & u.c. & n.d. & $\begin{array}{l}\text { Increased Triton } \\
\text { X-100-insoluble } \alpha \text {-syn } \\
\text { aggregates at day } 60 ; \\
\text { increased susceptibility to } \\
\text { mitochondria toxin at day } 60\end{array}$ & (63) \\
\hline \multicolumn{10}{|l|}{ ATP13A2 } \\
\hline $\begin{array}{l}\text { p.[Leu1059Arg] } \\
; \text {;[Leu1085fs] }\end{array}$ & $\mathrm{mDA}$ & $\begin{array}{l}\text { 2D-based }(45,47) \\
\sim 60 \% \mathrm{TH}^{+} / \\
\text {FOXA2 }^{+}\end{array}$ & FOXA2, TH (ICC) & No & Yes & n.d. & n.d. & $\begin{array}{c}\text { Increased detergent-insoluble } \\
\alpha \text {-syn aggregates at day } 90 ; \\
\text { lysosomal defects at day } 90\end{array}$ & (56) \\
\hline
\end{tabular}

sporadic PD hiPSC, although the authors did not assess the formation of $\alpha$-syn aggregates in these neurons (53).
Familial PD hiPSC-based model: SCNA mutations

Mutations in SCNA (also known as PARK1 and PARK4) gene, which encodes $\alpha$-syn, lead to autosomal dominant PD (1). The thioflavin S-positive and proteinase K-resistant $\alpha$-syn 
Table 1. Continued 2

\begin{tabular}{|c|c|c|c|c|c|c|c|c|c|}
\hline \multirow{2}{*}{$\begin{array}{l}\text { Genetic } \\
\text { mutation }\end{array}$} & \multirow{2}{*}{$\begin{array}{l}\text { Tested } \\
\text { model }\end{array}$} & \multirow{2}{*}{$\begin{array}{c}\text { Differentiation } \\
\text { protocol (ref.) }\end{array}$} & \multirow{2}{*}{$\begin{array}{l}\text { Differentiation } \\
\text { marker }\end{array}$} & \multirow{2}{*}{ Stimulus } & \multicolumn{2}{|c|}{ Aggregate } & \multirow{2}{*}{$\begin{array}{l}\text { Cell } \\
\text { death }\end{array}$} & \multirow{2}{*}{ Note } & \multirow{2}{*}{ Ref. } \\
\hline & & & & & WB & ICC & & & \\
\hline \multicolumn{10}{|l|}{$G B A$} \\
\hline $\begin{array}{l}\text { p.[Asn409Ser] } \\
\text {;[Leu29fs] }\end{array}$ & $\mathrm{mDA}$ & $\begin{array}{l}\text { 2D-based }(45,47) \\
\sim 60 \% \\
\mathrm{TH}^{+} / \text {FOXA2 }\end{array}$ & $\begin{array}{l}\text { LMX1A, FOXA2, } \\
\text { TH (ICC) }\end{array}$ & No & Yes & Yes & n.d. & $\begin{array}{l}\text { Increased detergent-insoluble } \\
\alpha \text {-syn aggregates at day } 90 ; \\
\text { thioflavin S-positive } \alpha \text {-syn } \\
\text { aggregates formation at day } \\
100 \text { or } 120 ; \text { increased low } \\
\text { expressing TH cells at day } \\
330 ; \text { lysosomal defects at day } \\
110,120 \text { or } 180 ; \text { reduced } \\
\text { aggregates formation by } 758 \\
\text { treatment at day } 120\end{array}$ & $\begin{array}{l}(51, \\
56)\end{array}$ \\
\hline \multirow[t]{2}{*}{$\begin{array}{l}\text { p. [Asn409Ser] } \\
;[=]\end{array}$} & $\mathrm{mDA}$ & $\begin{array}{l}\text { 2D-based }(45,47) \\
\sim 75 \% \mathrm{TH}^{+}\end{array}$ & $\begin{array}{l}\text { FOXA2, NURR1, } \\
\text { TH (qRT-PCR); } \\
\text { FOXA2, PITX3, } \\
\text { TH (ICC) }\end{array}$ & No & u.c. & n.d. & No & $\begin{array}{l}\text { Reduced ratio of tetramers and } \\
\text { related multimers, which resist } \\
\text { aggregation, to monomers at } \\
\text { day } 60-65\end{array}$ & (71) \\
\hline & & & & $\alpha-P F F$ & Yes & n.d. & Yes & $\begin{array}{l}\text { Increased detergent-insoluble } \\
\alpha \text {-syn aggregates at day } 73 ; \\
\text { increased pS129- } \alpha \text {-syn at day } \\
73 \text {; increased cell death at day } \\
73 \text { or } 79\end{array}$ & \\
\hline
\end{tabular}

2D, monolayer differentiation. 3D, neurosphere differentiation. 758, NCGC00188758. $\alpha$-PFF, $\alpha$-syn pre-formed fibril (72). BFCN, basal forebrain cholinergic neuron. cortical, cortical neuron. del, deletion. EB, embryonic body differentiation. EX, exon. fs, frameshift. GABAergic, GABAergic neuron. GLUergic, glutamatergic neuron. ICC, confirmed by immunocytochemistry. mDA, midbrain dopaminergic neuron. n.d., not determined. NPC, neuronal precursor cell. p., protein sequence. qRT-PCR, quantitative real-time PCR. u.c., unclear. WB, confirmed by western blotting.

aggregates were detected in the EB-derived 50 days old neurons differentiated from a familial PD hiPSC with a point mutation in SNCA gene (c. [157G $>A] ;[=] / p$. [Ala53Thr]; [=], also known as heterozygous A53T) (54). Moreover, the level of pS129- $\alpha$-syn was increased in the A53T neurons (54). Ryan et al. also reported an increase in thioflavin T-positive or Ser129-phosphorylated $\alpha$-syn aggregates formation and ROS production in the FP-derived 35 days old A53T mDA neurons, as compared to genetically corrected controls (55). The authors have further revealed the susceptibility of A53T mDA neurons to apoptosis induced by environmental pesticides, such as paraquat, maneb, and rotenone, compared to the controls (55). The presence of thioflavin S-positive $\alpha$-syn aggregates in the FP-derived 90 days old A53T mDA neurons has been demonstrated along with the reduction in aggregate formation upon treatment with $\beta$-glucocerebrosidase (GCase) activator, NCGC00188758 (56).

Copy number variations in SNCA locus are also linked to familial PD [duplication (5), triplication (4)]. Using a familial PD hiPSC with SNCA locus duplication, Prots et al. reported a significant increase in the level of $1 \%$ Triton $X-100 / 1 \%$ NP-40/1\% SDS-insoluble-and-8 M urea/5\% SDS-soluble $\alpha$-syn aggregates in the EB-derived PD neurons co-cultured with primary human cerebellar astrocytes, as compared to healthy controls (57). The authors performed sucrose density gradient centrifugation analysis to assess the composition of $\alpha$-syn aggregates and revealed an increase in the presence of $\alpha$-syn aggregation intermediates in the PD neurons (57). Other report exhibited the increased formation of filamentous $\alpha$-syn aggregates and cell death in the 70-90 days old cortical neurons differentiated from a familial PD hiPSC with SNCA locus triplication, as compared to genetically corrected controls (58). In addition, Tagliafierro et al. described an increase in the number of punctate $\alpha$-syn aggregates in the EB-derived 45-50 days old mDA neurons differentiated from a familial PD hiPSC with SNCA locus triplication compared to the controls (59). Moreover, the increment and the size of punctate $\alpha$-syn aggregates were more augmented in $>70$ days old PD mDA neurons, as compared to 45-50 days old neurons (59). The authors have additionally shown an increase in the formation and size of diffused $\alpha$-syn aggregates in $>70$ days old PD basal forebrain cholinergic neurons (BFCNs), which are primarily affected in DLB, compared to 45-50 days old neurons (59). Mazzulli et al. reported the detection of thioflavin S-positive $\alpha$-syn aggregates in the FP-derived 100 or 120 days old mDA neurons differentiated from a familial PD hiPSC with SNCA locus triplication $(51,56)$. In addition, the amount of pathogenic $\alpha$-syn detected by Syn 303 antibody was increased in the $1 \%$ Triton X-100-insoluble-and- $\%$ SDS-soluble fraction of 55-330 days old PD mDA neurons carrying an SNCA locus triplication than healthy hiPSC-derived mDA neurons (51). Another report described the increased level of 
pS129- $\alpha$-syn in the FP-derived 44-46 days old mDA neurons and revealed the increased susceptibility to neurotoxins in 34 days old mDA neurons, differentiated from a familial PD hiPSC with SNCA locus triplication compared to healthy hiPSCs (53).

\section{Familial PD hiPSC-based model: LRRK2 mutations}

Mutations in LRRK2 (also known as PARK8) gene, which encodes leucine-rich repeat serine/threonine-protein kinase 2, lead to autosomal dominant PD (60). Even though an attempt was made to analyze numerous articles which attempted to recapitulate PD phenotypes using PD hiPSCs with $L R R K 2$ mutations, none of the reports provided solid evidence showing $\alpha$-syn aggregation in patient-derived mDA neurons. Instead, one recently published paper reported an increase in $\alpha$-syn puncta in 28 days old PD astrocytes with a point mutation in the LRRK2 gene (c.[6055G >A]; [=]/p.[Gly2019Ser]; [=], also known as heterozygous G2019S), as compared to healthy astrocytes (61).

\section{Familial PD hiPSC-based model: PRKN mutations}

Mutations in PRKN (also known as PARK2) gene, which encodes E3 ubiquitin-protein ligase parkin, lead to autosomal recessive PD (60). Shaltouki et al. demonstrated the formation of tentative $\alpha$-syn aggregates in the EB-derived 28 days old mDA neurons differentiated from familial PD hiPSCs with various mutations in $P R K N$ gene; i.e., p.[Arg42Pro]; [=], p.[Arg275Trp];[=], p.[Arg42Pro];[EX3del], and p.[Asn52fs]; [EX3_4del] (62). On the other hand, Chung et al. reported a significant increase in the level of $1 \%$ Triton X-100-insolubleand-2\% SDS-soluble $\alpha$-syn aggregates in the FP-derived 60 days old $\mathrm{mDA}$ neurons differentiated from a PD hiPSC with same point mutations in both the PRKN alleles (c.[971delT]; [971delT]/p.[Val324Alafs*111];[Val324Alafs*111], also known as homozygous V324fs), as compared to healthy controls (63). Furthermore, the researchers provided evidence of the dependency on the type of differentiation protocol utilized; the FP-derived PD mDA neurons recapitulated PD phenotypes in vitro while the neural rosette-derived PD mDA neurons (40) did not recapitulate these phenotypes (63).

\section{Familial PD hiPSC-based model: PINK1 mutations}

Mutations in PINK1 (also known as PARK6) gene, which encodes serine/threonine-protein kinase PINK1 mitochondrial, lead to autosomal recessive PD (60). In the FP-derived 60 days old mDA neurons differentiated from a PD hiPSC with point mutations in the PINK1 gene (c. [1366C $>$ T]; [1366C $>$ T]/ p.[Gln456Ter];[Gln456Ter], also known as homozygous Q456X), the level of $1 \%$ Triton X-100-insoluble-and-2\% SDS-soluble $\alpha$-syn aggregates was significantly increased compared to healthy controls; however, it was not observed in the neural rosette-derived PD mDA neurons (63).

\section{Familial PD hiPSC-based model: $A T P 13 A 2$ mutations}

Mutations in ATP13A2 (also known as PARK9) gene, which encodes cation-transporting ATPase 13A2, lead to autosomal recessive PD (60). Mazzulli et al. reported that the amount of $1 \%$ Triton X-100-insoluble-and-2\% SDS-soluble $\alpha$-syn was increased in the FP-derived 90 days old mDA neurons differentiated from a familial PD hiPSC with point mutations in ATP13A2 gene (c.[3176T $>$ C];[3253delC]/p.[Leu1059Arg]; [Leu1085Trpfs*4], also known as heterozygous L1059R;L1085fs) compared to healthy control (56).

\section{PD-related hiPSC-based model: $\boldsymbol{G B A}$ mutations}

$G B A$ (also known as GBA1) gene, which encodes lysosomal acid glucosylceramidase (also known as $\beta$-glucocerebrosidase or GCase), has a critical role in glycolipid metabolism. Loss-of-function mutations in the GBA gene cause lysosomal defects via accumulation of lipid substrates in the lysosome that results in autosomal recessive Gaucher disease (GD) (64). Moreover, mutations in the GBA gene are well-known to increase the risk of developing PD and DLB (65-70). Using a GD hiPSC with mutations in the GBA gene (c.[1226A>G]; [84dupG]/p.[Asn409Ser];[Leu29Alafs*18], also known as heterozygous N409S;L29fs), Mazzulli et al. reported the detection of thioflavin S-positive $\alpha$-syn aggregates in the FP-derived 100 or 120 days old GD mDA neurons $(51,56)$. In addition, the authors demonstrated that the amount of pathogenic $\alpha$-syn detected by Syn303 antibody was increased in the $1 \%$ Triton $\mathrm{X}$-100-insoluble-and- $2 \%$ SDS-soluble fraction of the 90 days old GD mDA neurons compared to healthy hiPSC-derived mDA neurons (51).

Groundbreaking researches suggested that $\alpha$-syn exists as a helically folded tetramer in the physiological conditions and not in the form of natively unfolded monomer; the tetramer is rarely gets converted into the pathological aggregates $(23,24)$. A recent study has reported that the ratio of $\alpha$-syn tetramers and related multimers, which resist aggregation, to monomers was reduced in the FP-derived 60-65 days old mDA neurons differentiated from a PD hiPSC with a point mutation in GBA gene (c. [1226A $>G] ;[=] / p$. [Asn409Ser]; $[=]$, also known as heterozygous N409S) compared to healthy iPSCs; indirectly revealing that the level of $\alpha$-syn aggregates may be increased in the cells of the patient (71). The authors also revealed an increase in the levels of $1 \%$ Triton X-100-insoluble-and- $2 \%$ SDS-soluble $\alpha$-syn aggregates and pS129- $\alpha$-syn in the $\alpha$-syn pre-formed fibril [ $\alpha$-PFFs (72)]-treated FP-derived 73 days old PD mDA neurons compared to healthy controls (71).

\section{PERSPECTIVES}

It is feasible to reproduce the molecular pathology of $\alpha$-synucleinopathies without using any exogenous factors and by employing only hiPSC-based PD modeling system along with the patient's genetic background. However, only a handful of papers demonstrated success in recapitulating $\alpha$-syn 
aggregation in vitro, among a large number of PD hiPSC-based models (Table 1). It is hypothesized that there might be three potential underlying causes of this problem: i) insufficient aging, ii) incomplete differentiation protocol, and iii) lack of environmental cues.

Even though hiPSCs were generated by reprogramming of somatic cells from aged PD patients, their ages seemed to be reset to embryonic ages during the reprogramming process (73-76). Moreover, hiPSC-derived neurons, such as human mDA neurons, are largely regarded as embryonic stage neurons (73-76). Therefore, resolving an aging issue and/or a maturation issue is necessary to model late-onset diseases, such as PD and DLB. Apparently, the progerin-induced aging method could be used as one of the possible solutions (77).

We are constantly attempting to improve mDA neuronal differentiation protocols for modeling PD more complete, beyond EB-derived, feeder-dependent, neural rosette-derived, and FP-derived methods (Fig. 1). Very recently, the protocols to differentiate hPSCs or neuroepithelial stem cells into human midbrain organoids have been developed for the generation of more ideal human mDA neuronal models (78-80). These midbrain organoids will serve as a completer and more important biologically relevant cell sources for PD modeling.

Environmental factors may cause PD along with genetic factors. Peng et al. demonstrated that environmental factors, such as neurotoxins, together with genetic mutations in PD in the same animal, synergistically accelerate age-related neurodegeneration (81). Similarly, only after treatment of $\alpha-P F F s, P D-r e l e v a n t$ phenotypes including increased levels of detergent-insoluble $\alpha$-syn aggregates and pS129- $\alpha$-syn were detected in $\mathrm{mDA}$ neurons differentiated from PD hiPSC with a GBA mutation (p.N409S) (71). These results suggest that the addition of environmental factors to the PD iPSC models might be the key to reveal the hidden phenotypes.

\section{ACKNOWLEDGEMENTS}

This work was supported by the National Research Foundation of Korea (NRF) grants funded by different governments of Korea (Ministry of Education, NRF-2017R1A6A3A03010524; Ministry of Science and ICT, NRF-2018R1C1B5045395) and by the research fund of Hanyang University (HY-2018). I sincerely apologize to colleagues whose work has not been cited in this review due to space limitations.

\section{CONFLICTS OF INTEREST}

The author has no conflicting interests.

\section{REFERENCES}

1. Olanow CW and Tatton WG (1999) Etiology and pathogenesis of Parkinson's disease. Annu Rev Neurosci $22,123-144$

2. Dauer W and Przedborski S (2003) Parkinson's disease: mechanisms and models. Neuron 39, 889-909

3. Polymeropoulos MH, Lavedan C, Leroy E et al (1997) Mutation in the alpha-synuclein gene identified in families with Parkinson's disease. Science 276, 2045-2047

4. Singleton $A B$, Farrer $M$, Johnson J et al (2003) alphaSynuclein locus triplication causes Parkinson's disease. Science 302, 841

5. Chartier-Harlin MC, Kachergus J, Roumier C et al (2004) Alpha-synuclein locus duplication as a cause of familial Parkinson's disease. Lancet 364, 1167-1169

6. Lee VM and Trojanowski JQ (2006) Mechanisms of Parkinson's disease linked to pathological alpha-synuclein: new targets for drug discovery. Neuron 52, 33-38

7. Fujiwara $H$, Hasegawa $M$, Dohmae $N$ et al (2002) alpha-Synuclein is phosphorylated in synucleinopathy lesions. Nat Cell Biol 4, 160-164

8. Jackson-Lewis V, Blesa J and Przedborski S (2012) Animal models of Parkinson's disease. Parkinsonism Relat Disord 18 Suppl 1, S183-185

9. Koprich JB, Kalia LV and Brotchie JM (2017) Animal models of alpha-synucleinopathy for Parkinson disease drug development. Nat Rev Neurosci 18, 515-529

10. Brennand KJ, Simone A, Jou J et al (2011) Modelling schizophrenia using human induced pluripotent stem cells. Nature 473, 221-225

11. Dimos JT, Rodolfa KT, Niakan KK et al (2008) Induced pluripotent stem cells generated from patients with ALS can be differentiated into motor neurons. Science 321, 1218-1221

12. Laflamme MA, Chen KY, Naumova AV et al (2007) Cardiomyocytes derived from human embryonic stem cells in pro-survival factors enhance function of infarcted rat hearts. Nat Biotechnol 25, 1015-1024

13. Li XJ, Du ZW, Zarnowska ED et al (2005) Specification of motoneurons from human embryonic stem cells. Nat Biotechnol 23, 215-221

14. Maroof AM, Brown K, Shi SH, Studer L and Anderson SA (2010) Prospective isolation of cortical interneuron precursors from mouse embryonic stem cells. J Neurosci 30, 4667-4675

15. Cunningham M, Cho JH, Leung A et al (2014) hPSC-derived maturing GABAergic interneurons ameliorate seizures and abnormal behavior in epileptic mice. Cell Stem Cell 15, 559-573

16. Joseph NM and Morrison SJ (2005) Toward an understanding of the physiological function of Mammalian stem cells. Dev Cell 9, 173-183

17. Saha K and Jaenisch R (2009) Technical challenges in using human induced pluripotent stem cells to model disease. Cell Stem Cell 5, 584-595

18. Li H, Jiang H, Zhang B and Feng J (2018) Modeling Parkinson's Disease Using Patient-specific Induced Pluripotent Stem Cells. J Parkinsons Dis 8, 479-493

19. Sison SL, Vermilyea SC, Emborg ME and Ebert AD (2018) Using Patient-Derived Induced Pluripotent Stem Cells to Identify Parkinson's Disease-Relevant Phenotypes. Curr Neurol Neurosci Rep 18, 84

20. Ueda K, Fukushima H, Masliah E et al (1993) Molecular cloning of cDNA encoding an unrecognized component of amyloid in Alzheimer disease. Proc Natl Acad Sci U S 
A $90,11282-11286$

21. Weinreb PH, Zhen W, Poon AW, Conway KA and Lansbury PT Jr (1996) NACP, a protein implicated in Alzheimer's disease and learning, is natively unfolded. Biochemistry 35, 13709-13715

22. Davidson WS, Jonas A, Clayton DF and George JM (1998) Stabilization of alpha-synuclein secondary structure upon binding to synthetic membranes. J Biol Chem 273, 9443-9449

23. Bartels T, Choi JG and Selkoe DJ (2011) alpha-Synuclein occurs physiologically as a helically folded tetramer that resists aggregation. Nature 477, 107-110

24. Wang W, Perovic I, Chittuluru J et al (2011) A soluble alpha-synuclein construct forms a dynamic tetramer. Proc Natl Acad Sci U S A 108, 17797-17802

25. Dettmer U, Newman AJ, Soldner F et al (2015) Parkinsoncausing alpha-synuclein missense mutations shift native tetramers to monomers as a mechanism for disease initiation. Nat Commun 6, 7314

26. Tong J, Wong H, Guttman M et al (2010) Brain alpha-synuclein accumulation in multiple system atrophy, Parkinson's disease and progressive supranuclear palsy: a comparative investigation. Brain 133, 172-188

27. Winklhofer KF, Tatzelt J and Haass C (2008) The two faces of protein misfolding: gain- and loss-of-function in neurodegenerative diseases. EMBO J 27, 336-349

28. McCann $\mathrm{H}$, Stevens $\mathrm{CH}$, Cartwright $\mathrm{H}$ and Halliday GM (2014) alpha-Synucleinopathy phenotypes. Parkinsonism Relat Disord 20 Suppl 1, S62-67

29. Savica R, Grossardt BR, Bower JH, Ahlskog JE and Rocca WA (2013) Incidence and pathology of synucleinopathies and tauopathies related to parkinsonism. JAMA Neurol 70, 859-866

30. Oueslati A, Fournier M and Lashuel HA (2010) Role of post-translational modifications in modulating the structure, function and toxicity of alpha-synuclein: implications for Parkinson's disease pathogenesis and therapies. Prog Brain Res 183, 115-145

31. Anderson JP, Walker DE, Goldstein JM et al (2006) Phosphorylation of Ser-129 is the dominant pathological modification of alpha-synuclein in familial and sporadic Lewy body disease. J Biol Chem 281, 29739-29752

32. Chen L and Feany MB (2005) Alpha-synuclein phosphorylation controls neurotoxicity and inclusion formation in a Drosophila model of Parkinson disease. Nat Neurosci 8, 657-663

33. Yamada $M$, Iwatsubo $T$, Mizuno $Y$ and Mochizuki $H$ (2004) Overexpression of alpha-synuclein in rat substantia nigra results in loss of dopaminergic neurons, phosphorylation of alpha-synuclein and activation of caspase-9: resemblance to pathogenetic changes in Parkinson's disease. J Neurochem 91, 451-461

34. Takahashi M, Kanuka H, Fujiwara $\mathrm{H}$ et al (2003) Phosphorylation of alpha-synuclein characteristic of synucleinopathy lesions is recapitulated in alpha-synuclein transgenic Drosophila. Neurosci Lett 336, 155-158

35. Oueslati A (2016) Implication of Alpha-Synuclein Phosphorylation at S129 in Synucleinopathies: What Have We Learned in the Last Decade? J Parkinsons Dis 6, 39-51
36. Tenreiro S, Eckermann K and Outeiro TF (2014) Protein phosphorylation in neurodegeneration: friend or foe? Front Mol Neurosci 7, 42

37. Arenas E, Denham M and Villaescusa JC (2015) How to make a midbrain dopaminergic neuron. Development 142, 1918-1936

38. Park CH, Minn YK, Lee JY et al (2005) In vitro and in vivo analyses of human embryonic stem cell-derived dopamine neurons. J Neurochem 92, 1265-1276

39. Zeng X, Cai J, Chen J et al (2004) Dopaminergic differentiation of human embryonic stem cells. Stem Cells 22, 925-940

40. Perrier AL, Tabar V, Barberi T et al (2004) Derivation of midbrain dopamine neurons from human embryonic stem cells. Proc Natl Acad Sci U S A 101, 12543-12548

41. Sonntag KC, Pruszak J, Yoshizaki T, van Arensbergen J Sanchez-Pernaute R and Isacson O (2007) Enhanced yield of neuroepithelial precursors and midbrain-like dopaminergic neurons from human embryonic stem cells using the bone morphogenic protein antagonist noggin. Stem Cells 25, 411-418

42. Roy NS, Cleren C, Singh SK, Yang L, Beal MF and Goldman SA (2006) Functional engraftment of human ES cell-derived dopaminergic neurons enriched by coculture with telomerase-immortalized midbrain astrocytes. Nat Med 12, 1259-1268

43. Kim BK, Kim SE, Shim JH et al (2006) Neurogenic effect of vascular endothelial growth factor during germ layer formation of human embryonic stem cells. FEBS Lett 580, 5869-5874

44. Swistowski A, Peng J, Han Y, Swistowska AM, Rao MS and Zeng $X$ (2009) Xeno-free defined conditions for culture of human embryonic stem cells, neural stem cells and dopaminergic neurons derived from them. PLoS One 4, e6233

45. Chambers SM, Fasano CA, Papapetrou EP, Tomishima M Sadelain M and Studer L (2009) Highly efficient neural conversion of human ES and iPS cells by dual inhibition of SMAD signaling. Nat Biotechnol 27, 275-280

46. Fasano CA, Chambers SM, Lee G, Tomishima MJ and Studer L (2010) Efficient derivation of functional floor plate tissue from human embryonic stem cells. Cell Stem Cell 6, 336-347

47. Kriks S, Shim JW, Piao J et al (2011) Dopamine neurons derived from human ES cells efficiently engraft in animal models of Parkinson's disease. Nature 480, 547-551

48. Chambers SM, Qi Y, Mica Y et al (2012) Combined small-molecule inhibition accelerates developmental timing and converts human pluripotent stem cells into nociceptors. Nat Biotechnol 30, 715-720

49. Soldner F, Hockemeyer D, Beard C et al (2009) Parkinson's disease patient-derived induced pluripotent stem cells free of viral reprogramming factors. Cell 136, 964-977

50. Baba M, Nakajo S, Tu PH et al (1998) Aggregation of alpha-synuclein in Lewy bodies of sporadic Parkinson's disease and dementia with Lewy bodies. Am J Pathol 152, 879-884

51. Mazzulli JR, Zunke F, Isacson O, Studer L and Krainc D (2016) alpha-Synuclein-induced lysosomal dysfunction 
occurs through disruptions in protein trafficking in human midbrain synucleinopathy models. Proc Natl Acad Sci U S A 113, 1931-1936

52. Giasson BI, Duda JE, Murray IV et al (2000) Oxidative damage linked to neurodegeneration by selective alpha-synuclein nitration in synucleinopathy lesions. Science 290, 985-989

53. Lin L, Goke J, Cukuroglu E, Dranias MR, VanDongen AM and Stanton LW (2016) Molecular Features Underlying Neurodegeneration Identified through In Vitro Modeling of Genetically Diverse Parkinson's Disease Patients. Cell Rep 15, 2411-2426

54. Kouroupi G, Taoufik E, Vlachos IS et al (2017) Defective synaptic connectivity and axonal neuropathology in a human iPSC-based model of familial Parkinson's disease. Proc Natl Acad Sci U S A 114, E3679-E3688

55. Ryan SD, Dolatabadi N, Chan SF et al (2013) Isogenic human iPSC Parkinson's model shows nitrosative stressinduced dysfunction in MEF2-PGC1alpha transcription. Cell 155, 1351-1364

56. Mazzulli JR, Zunke F, Tsunemi T et al (2016) Activation of beta-Glucocerebrosidase Reduces Pathological alphaSynuclein and Restores Lysosomal Function in Parkinson's Patient Midbrain Neurons. J Neurosci 36, 7693-7706

57. Prots I, Grosch J, Brazdis RM et al (2018) alpha-Synuclein oligomers induce early axonal dysfunction in human iPSC-based models of synucleinopathies. Proc Natl Acad Sci U S A 115, 7813-7818

58. Ludtmann MHR, Angelova PR, Horrocks MH et al (2018) alpha-synuclein oligomers interact with ATP synthase and open the permeability transition pore in Parkinson's disease. Nat Commun 9, 2293

59. Tagliafierro L, Zamora ME and Chiba-Falek O (2019) Multiplication of the SNCA locus exacerbates neuronal nuclear aging. Hum Mol Genet 28, 407-421

60. Klein C and Westenberger A (2012) Genetics of Parkinson's disease. Cold Spring Harb Perspect Med 2, a008888

61. di Domenico A, Carola G, Calatayud C et al (2019) Patient-Specific iPSC-Derived Astrocytes Contribute to Non-Cell-Autonomous Neurodegeneration in Parkinson's Disease. Stem Cell Reports 12, 213-229

62. Shaltouki A, Sivapatham R, Pei Y et al (2015) Mitochondrial alterations by PARKIN in dopaminergic neurons using PARK2 patient-specific and PARK2 knockout isogenic iPSC lines. Stem Cell Reports 4, 847-859

63. Chung SY, Kishinevsky S, Mazzulli JR et al (2016) Parkin and PINK1 Patient iPSC-Derived Midbrain Dopamine Neurons Exhibit Mitochondrial Dysfunction and alphaSynuclein Accumulation. Stem Cell Reports 7, 664-677

64. Hruska KS, LaMarca ME, Scott CR and Sidransky E (2008) Gaucher disease: mutation and polymorphism spectrum in the glucocerebrosidase gene (GBA). Hum Mutat 29, 567-583

65. Nalls MA, Duran R, Lopez G et al (2013) A multicenter study of glucocerebrosidase mutations in dementia with Lewy bodies. JAMA Neurol 70, 727-735

66. Sidransky E, Nalls MA, Aasly JO et al (2009) Multicenter analysis of glucocerebrosidase mutations in Parkinson's disease. N Engl J Med 361, 1651-1661
67. Eblan MJ, Walker JM and Sidransky E (2005) The glucocerebrosidase gene and Parkinson's disease in Ashkenazi Jews. N Engl J Med 352, 728-731; author reply 728-731

68. Goker-Alpan O, Schiffmann R, LaMarca ME, Nussbaum RL, Mclnerney-Leo A and Sidransky E (2004) Parkinsonism among Gaucher disease carriers. J Med Genet 41, 937-940

69. Halperin A, Elstein D and Zimran A (2006) Increased incidence of Parkinson disease among relatives of patients with Gaucher disease. Blood Cells Mol Dis 36, 426-428

70. Lwin A, Orvisky E, Goker-Alpan O, LaMarca ME and Sidransky E (2004) Glucocerebrosidase mutations in subjects with parkinsonism. Mol Genet Metab 81, 70-73

71. Kim S, Yun SP, Lee S et al (2018) GBA1 deficiency negatively affects physiological alpha-synuclein tetramers and related multimers. Proc Natl Acad Sci U S A 115, 798-803

72. Luk KC, Song C, O'Brien P et al (2009) Exogenous alpha-synuclein fibrils seed the formation of Lewy body-like intracellular inclusions in cultured cells. Proc Natl Acad Sci U S A 106, 20051-20056

73. Soria-Valles C and Lopez-Otin C (2016) iPSCs: On the Road to Reprogramming Aging. Trends Mol Med 22, 713-724

74. Zeltner N and Studer L (2015) Pluripotent stem cell-based disease modeling: current hurdles and future promise. Curr Opin Cell Biol 37, 102-110

75. Vera E and Studer L (2015) When rejuvenation is a problem: challenges of modeling late-onset neurodegenerative disease. Development 142, 3085-3089

76. Studer L, Vera E and Cornacchia D (2015) Programming and Reprogramming Cellular Age in the Era of Induced Pluripotency. Cell Stem Cell 16, 591-600

77. Miller JD, Ganat YM, Kishinevsky S et al (2013) Human iPSC-based modeling of late-onset disease via progerin-induced aging. Cell Stem Cell 13, 691-705

78. Bolognin S, Fossepre M, Qing X et al (2019) 3D Cultures of Parkinson's Disease-Specific Dopaminergic Neurons for High Content Phenotyping and Drug Testing. Adv Sci (Weinh) 6, 1800927

79. Jo J, Xiao Y, Sun AX et al (2016) Midbrain-like Organoids from Human Pluripotent Stem Cells Contain Functional Dopaminergic and Neuromelanin-Producing Neurons. Cell Stem Cell 19, 248-257

80. Monzel AS, Smits LM, Hemmer K et al (2017) Derivation of Human Midbrain-Specific Organoids from Neuroepithelial Stem Cells. Stem Cell Reports 8, 1144-1154

81. Peng J, Oo ML and Andersen JK (2010) Synergistic effects of environmental risk factors and gene mutations in Parkinson's disease accelerate age-related neurodegeneration. J Neurochem 115, 1363-1373

82. Havlicek S, Kohl Z, Mishra HK et al (2014) Gene dosage-dependent rescue of HSP neurite defects in SPG4 patients' neurons. Hum Mol Genet 23, 2527-2541

83. Tagliafierro L, Glenn OC, Zamora ME et al (2017) Genetic analysis of alpha-synuclein 3' untranslated region and its corresponding microRNAs in relation to Parkinson's disease compared to dementia with Lewy bodies. Alzheimers Dement 13, 1237-1250 
84. Hu Y, Qu ZY, Cao SY et al (2016) Directed differentiation of basal forebrain cholinergic neurons from human pluripotent stem cells. J Neurosci Methods 266, 42-49

85. Crompton LA, Byrne ML, Taylor H et al (2013) Stepwise, non-adherent differentiation of human pluripotent stem cells to generate basal forebrain cholinergic neurons via hedgehog signaling. Stem Cell Res 11, 1206-1221

86. Serio A, Bilican B, Barmada SJ et al (2013) Astrocyte pathology and the absence of non-cell autonomy in an induced pluripotent stem cell model of TDP-43 proteinopathy. Proc Natl Acad Sci U S A 110, 4697-4702 